\title{
Perspectivas acerca do conceito de saúde mental: análise das produções científicas brasileiras
}

\author{
Perspectives on the mental health concept: \\ analysis of Brazilian scientific productions
}

Vírnia Ponte Alcântara (https://orcid.org/0000-0002-8385-8795) ${ }^{1}$

Camilla Araújo Lopes Vieira (https://orcid.org/0000-0003-1706-3772) ${ }^{1}$

Samara Vasconcelos Alves (https://orcid.org/0000-0002-5452-2336) ${ }^{1}$

${ }^{1}$ Universidade Federal do

\begin{abstract}
The frequent use of the term mental health is noticeable in the field of health. It is employed in legislations, public policies, manuals, media, and designates health services. This recurrence raises questions about this concept. Therefore, this study aims to analyze it from the understanding of its theoretical models, showed by the excerpts of the scientific production. We adopted the evolutionary Concept Analysis model developed by Rodgers. Data was collected in papers available in the Scopus and Web of Science databases. We included high-impact factor papers published between 2013 and 2017, written in Portuguese and English, whose country of origin was Brazil. Duplicate and systematic and integrative reviews were excluded. Data were collected based on repeated patterns, in the following categories: a) context; b) substitute terms; c) attributes; $d$ ) antecedents and consequents; e) implications and hypotheses. We observed that mental health is a cross-cutting theme and is influenced by many knowledge areas. Mental health is related to a way of understanding health and mental illness from the paradigms of psychiatry and the Psychiatric Reform.
\end{abstract}

Key words Mental health, Concept formation, Psychiatry, Mental health assistance
Resumo No campo da saúde é perceptível o uso frequente do termo saúde mental, que é utilizado em legislações, políticas governamentais, manuais, além de designar serviços de saúde. Essa reincidência provoca curiosidade acerca do conceito de saúde mental. Logo, o objetivo é analisá-lo a partir da compreensão de seus modelos teóricos, construídos pelo recorte da produção científica. Foi utilizado o modelo evolucionário da Análise de Conceito desenvolvido por Rodgers. O levantamento de dados se deu em artigos disponiveis nas bases Scopus e Web of Science. Foram incluídos os artigos com alto Fator de Impacto, publicados entre 2013 e 2017, escritos em português e inglês, cujo país de origem fosse o Brasil. Foram excluídos os trabalhos duplicados e os de revisão integrativa e sistemática. Os dados foram coletados com base em padrões repetidos, nas categorias: a) contexto; b) termos substitutos; c) atributos; $d$ ) antecedentes e consequentes; e) implicações e hipóteses. Percebeu-se que saúde mental é um tema transversal a várias disciplinas e sofre influência de muitos saberes. Saúde mental está relacionada a um modo de entender a saúde e a doença psíquica a partir dos paradigmas da psiquiatria e da reforma psiquiátrica.

Palavras-chave Saúde mental, Formação de conceito, Psiquiatria, Assistência em saúde mental 


\section{Introdução}

Em uma aproximação com o campo da saúde, torna-se perceptível o uso frequente do termo saúde mental. Ele é utilizado em legislações ou políticas governamentais, como designação de serviços da saúde, também aparece em manuais, em artigos científicos, em livros, nos meios de comunicação, além de ser referido pela comunidade em geral. Ainda assim, essa constante e curiosa repetição não indica que exista um consenso sobre o que, de fato, signifique saúde mental.

Alguns dos mais importantes autores que se debruçaram na gênese dessa temática, como Canguilhem $^{1}$ e Foucault ${ }^{2}$, o fizeram através da construção de certa oposição entre saúde e doença e como essas duas categorias foram sendo constituídas discursivamente, ao longo do tempo, em suas dimensões culturais, sociais, políticas e econômicas. Tal oposição indica as marcas das mudanças radicais de ordem científica, sustentadas por um pragmatismo desenfreado que coloca a verdade e a razão na gênese do conhecimento. Uma operação realizada a partir dos infindáveis desdobramentos cartesianos instaurados pelo discurso tecnológico que insiste em separar o corpo (somático) da mente (psíquico) e que persistem hegemônicos no raciocínio biomédico.

À vista disso, dois modos de entendimento da saúde e doença se destacam: 1) a compreensão positivista, pragmática, baseada nas ciências empíricas - biologia, física e química -, que considera a saúde e a doença através da bioestatística e do selecionismo. Nessa perspectiva, saúde e doença estão relacionadas à normalidade. Logo, a anormalidade seria um desvio estatístico entre o atípico e o funcionamento normal do organismo, comparando o funcionamento de cada indivíduo com o funcionamento geral da espécie, em larga escala ${ }^{3}$; 2) a compreensão subjetivista, que pensa a saúde e a doença por meio de um universo linguístico e genealógico, relacionados à cultura, gramática, epistemologia, antropologia e história. Saúde e doença não são vistas como um desvio na norma, mas como uma construção mutável, com caráter subjetivo e intencional, conhecidas por meio da descrição dos fenômenos ${ }^{3}$. Essa compreensão se divide em duas outras: 2.a) as que percebem a saúde como um valor externo, baseado nas regras de uma determinada comunidade - que definem aquilo que é a doença; 2.b) e as que percebem como valor interno, baseado naquilo que os indivíduos descrevem como percepção da própria incapacidade - a experiência de adoecimento ${ }^{3}$.
Decorre disso um esforço para conceber a patologia mental pelo viés orgânico. $\mathrm{O}$ impacto do encontro com a loucura fez o campo epistemológico atualizar a divisão das ciências naturais e ciências sociais. Assim, a loucura foi sendo deslocada da desrazão ${ }^{2}$ à doença mental, quando a psiquiatria assume o cuidado dos loucos e desenvolve seu saber sobre a alienação.

Esse deslocamento se deu a partir do confinamento da loucura no manicômio. A institucionalização asilar, pelas vias da internação no hospital psiquiátrico, garantiu que a doença mental fosse submetida enquanto objeto da intervenção da medicina mental. Dessa forma, a loucura, capturada pela clínica psiquiátrica e pelo discurso médico científico, se transformou em alienação e depois em doença mental por meio do diagnóstico e do tratamento ${ }^{4}$. Esse tratamento garantido pela psiquiatria era pedagógico-disciplinar e intencionava a cura através da reeducação moral capaz de corrigir o distúrbio das paixões, ou seja, fazer com que o louco sujeitasse seu delírio à sua própria vontade para voltar à razão ${ }^{4}$. Uma medida terapêutica pautada no isolamento que era compreendida como um direito e não uma violação.

Apenas com o fim da Segunda Guerra Mundial, com a popularização de terapias psicológicas e o com advento dos psicofármacos, que se reconheceu o isolamento do doente mental nas instituições como um modelo de tratamento desumano e segregante ${ }^{5}$. Tratamento esse que cerceava a liberdade e autonomia desses indivíduos submetendo-os às condições de intenso sofrimento. Essa compreensão culminou em movimentos de reforma psiquiátrica que foram se levantando pelo mundo todo, principalmente em países como Itália, França, Inglaterra, Estados Unidos e Canadá, com tempos e características distintas. Apenas em um segundo momento, países como a Espanha e o Brasil também reformaram sua assistência psiquiátrica ${ }^{6}$.

No Brasil, a reforma iniciou durante a década de 1970, durante o processo de redemocratização do país, ocorrendo juntamente com a reforma sanitária. Foi protagonizada pelo movimento da Luta antimanicomial, composto por trabalhadores, familiares, usuários, artistas e políticos envolvidos com as transformações dos cuidados com os doentes mentais que culminou no modelo de atenção psicossocial, conforme Amarante e Lanceti ${ }^{7}$.

Apesar de tantas lutas para a construção desse novo modelo de atenção, a compreensão de saúde e doença, proposta pela discursividade cientí- 
fica, acaba adquirindo muita força no campo da atenção psicossocial. A saúde é percebida como um ideal, e a doença é como um problema que precisa ser localizado, descrito e medido. A doença deve ser diagnosticada e eliminada sem perca de tempo. Na seara do pragmatismo desenfreado, o que resta é rechaçado, sem valor ${ }^{7}$. Uma tentativa da ciência de nada querer saber sobre as falhas e negar o mal-estar inerente à nossa própria constituição.

Um processo de psiquiatrização da vida, de medicalização das subjetividades e dos sujeitos, cujo sinal mais evidente é a utilização do reducionismo classificatório dos manuais de diagnóstico psiquiátrico - os DSM's e CID's ${ }^{8}(\mathrm{p} .45)$.

Importante lembrar que a Organização Mundial da Saúde (OMS), dissemina em seus relatórios um conceito de saúde mental entendido como "[...] um estado de bem-estar no qual o indivíduo perceba as suas próprias capacidades, possa lidar com as tensões normais da vida, possa trabalhar de forma produtiva e frutífera e possa contribuir para a sua comunidade" . O termo "bem-estar" também aparece no conceito de saúde defendido pela OMS, como completo bem-estar físico, psíquico e social. Esse é o discurso da higiene, que fala Canguilhem ${ }^{10}$, quando diz que a disciplina médica tradicional, para regulamentar a vida dos indivíduos, se transveste de uma ambição sociopolítico-médica.

Diante de tantas transformações implicando as compreensões acerca da saúde, o que se percebe é uma verdadeira polissemia envolvendo o conceito de saúde mental e o desenvolvimento de mais de um modelo teórico para defini-la. Almeida Filho et al. ${ }^{11}$, em sua tentativa de conceituar saúde mental, distinguiram duas formas de definição: por meio da vertente narrativa, que descreve a etnografia ou retórica popular; ou pela vertente interpretativa, que é uma maneira de uma comunidade identificar e interpretar suas práticas partindo de elementos sociais, políticos, econômicos, culturais, individuais ou familiares. A interpretação de segundo nível seria aquela que tenta traduzir a linguagem científica, que tende à universalidade, enquanto que é produzida e produtora de práticas e saberes ${ }^{11}$.

Deste modo, dada a importância da interpretação da ciência sobre os fenômenos e a dificuldade de conceituar saúde mental, o objetivo dessa pesquisa é analisar o conceito de saúde mental a partir da compreensão de seus modelos teóricos, do ponto de vista da produção científica, tendo por recorte as pesquisas brasileiras.

\section{Metodologia}

O método utilizado foi o modelo evolucionário da Análise de Conceito desenvolvido por Rodgers $^{12}$. Para o autor, os conceitos são dinâmicos, amplos e estão associados ao contexto de seu uso. Conceitos são entendidos como uma reverberação dos fenômenos, eles têm caráter indutivo, descritivo, histórico e devem ser atribuídos com rigor sistemático. Sua construção se dá a partir da identificação e agrupamento de características e qualidades de um determinado fenômeno. Essas características são associadas em uma classe abstrata cuja expressão mantém um significado ${ }^{13}$.

$\mathrm{O}$ método foi realizado em cinco diferentes etapas $^{13}$ : a primeira foi a identificação do conceito significante com o qual se desejava trabalhar - no caso, saúde mental; a segunda etapa foi realizar o levantamento de dados para a pesquisa. Poderiam ser incluídos livros, documentos, leis, artigos, arquivos bibliográficos, assim como obras de arte, dança, música, cinema ou escultura. Devido o recorte da pesquisa, optou-se por utilizar apenas artigos científicos disponíveis nas bases de dados Scopus e Web of Science, contendo o descritor "Saúde Mental".

A terceira etapa consistiu na coleta de dados, em que foram observados os critérios de inclusão e exclusão. Foram incluídos artigos científi$\cos$ (pesquisas de campo, pesquisas bibliográficas documentais e ensaios); publicados nos últimos cinco anos (2013-2017); escritos em português e inglês; cujo país de origem fosse o Brasil - para que houvesse o recorte contextual relacionado à realidade brasileira; com maior Fator de Impacto (FI) na comunidade científica (utilizando o contador de citações da base Scielo - SciELO Citation Index). Foram excluídos os artigos que se repetiam e os trabalhos de revisão sistemática e revisão integrativa de artigos.

O Fator de Impacto (FI) é um ranking de periódicos científicos baseado na análise cienciométrica dos estudos, ou seja, disponibiliza uma contagem do número de vezes em que o artigo foi citado, que é o h-index ou índice-h, revelando os artigos com maior indicador bibliométrico. $\mathrm{O}$ FI é o meio mais utilizado para mensurar o impacto dos periódicos na comunidade científica, no entanto é importante demarcar que o ranking nem sempre aponta os estudos de maior qualidade, os mais inovadores ou os trabalhos mais relevantes da carreira de pesquisadores, apenas aqueles de maior impacto ${ }^{14-17}$.

Após a aplicação dos critérios de inclusão, foram encontrados 64 artigos na base Scopus e 
891 artigos na base Web of Science (de SciELO Citation Index). Aplicando o Fator de Impacto, foram escolhidos arbitrariamente os 20 artigos mais citados de cada base, totalizando 40 trabalhos para análise dos títulos e resumos e aplicação dos critérios de exclusão. Foram excluídos os artigos que se repetiam e os trabalhos de revisão sistemática e revisão integrativa de artigos. Desse modo restaram 31 artigos para análise. A escolha arbitrária do número de trabalhos selecionados se deu por considerar um quantitativo adequado para análise, entendendo que adicionar mais trabalhos desconsideraria a intenção de observar os de maior impacto (Figura 1).

A quarta etapa consistiu na análise dos textos com base nos atributos relacionados ao termo saúde mental. Esses atributos ${ }^{18}$, considerados na análise dos dados coletados, foram questionamentos que poderiam identificar padrões repetidos nos textos, como: a) o contexto no qual se insere o conceito em questão - que pode se referir às disciplinas com as quais o conceito se relaciona, ou as bases teóricas e epistemológicas associadas, ou os grupos que se relacionam com o conceito, dentre outros ${ }^{18}$; b) Termos substitutos - que são as palavras que se relacionam ou expressam as mesmas ideias do conceito $^{18}$; c) os atributos do conceito em pauta - que são as palavras e as expressões que, repetidamente, são empregadas para descrever as características do conceito $^{18}$; d) os antecedentes e consequentes do conceito - em que os antecedentes são os episódios, cenários ou fenômenos que antecedem o conceito e facilitam a compreensão do contexto social e os consequentes são os episódios que resultam do uso do conceito $^{18}$; e) as implicações e hipóteses do conceito - tendo em vista que o conceito não é estático, esse método de pesquisa desaconselha apresentar uma abordagem conclusiva, mas sim indagar e apresentar hipóteses para pesquisas futuras ${ }^{18}$.

\section{Resultados}

Dos 31 trabalhos selecionados, 14 foram mencionados na base Scopus, 12 na base Web of Sciencee cinco estavam em ambas as bases, embora essas plataformas de pesquisa apresentassem diferentes contagens das citações de cada artigo. $\mathrm{O}$ artigo mais citado foi mencionado 18 vezes e os menos citados foram mencionados quatro vezes cada (Quadro 1).

Com relação aos periódicos, os que mais apareceram foram a revista "Ciência \& Saúde Coletiva", com oito publicações; e os "Cadernos de Saúde Pública”, com cinco publicações, as demais aparecem em menor frequência. Com relação ao idioma, 19 artigos foram publicados em português, cinco artigos foram publicados em inglês e sete estavam publicados em ambos os idiomas. Sobre o ano de publicação, 14 trabalhos eram de 2013, 14 trabalhos eram de 2014, dois trabalhos eram de 2015e apenas um era de 2016, o que pode ser explicado tendo em vista que quanto mais antigo o trabalho, maior impacto ele tem por conta do tempo de circulação, sendo essa uma limitação prevista da pesquisa ${ }^{16,17}$ (Quadro 1).

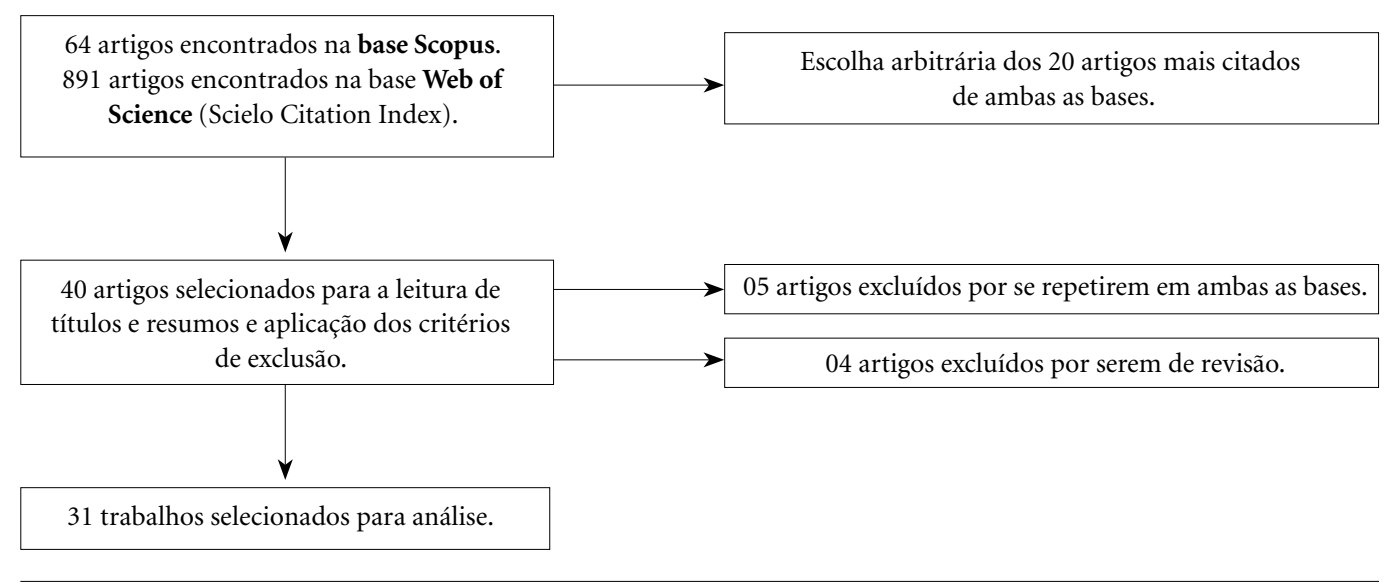

Figura 1. Fluxograma dos artigos selecionados para análise após critérios de inclusão e exclusão. 
Quadro 1. Caracterização dos estudos analisados.

\begin{tabular}{|c|c|c|c|c|c|}
\hline $\begin{array}{l}\text { No de } \\
\text { citações }\end{array}$ & $\begin{array}{l}\text { Base de } \\
\text { Dados }\end{array}$ & Título do artigo & Autor/Ano & Periódico & Idioma \\
\hline 18 & $\begin{array}{l}\text { Web of } \\
\text { Science }\end{array}$ & $\begin{array}{l}\text { Estudo multicêntrico brasileiro sobre } \\
\text { transtornos mentais comuns na } \\
\text { atenção primária: prevalência e fatores } \\
\text { sociodemográficos relacionados }\end{array}$ & $\begin{array}{l}\text { Gonçalves } \\
\text { et al. } \\
(2014)^{19}\end{array}$ & $\begin{array}{l}\text { Cadernos de } \\
\text { Saúde Pública }\end{array}$ & Inglês \\
\hline $\begin{array}{l}15(\mathrm{~S}) \\
8(\mathrm{WoS})\end{array}$ & $\begin{array}{l}\text { Scopus e } \\
\text { Web of } \\
\text { Science }\end{array}$ & $\begin{array}{l}\text { Discriminação de idade, classe e raça: Suas } \\
\text { interações e associações com saúde mental } \\
\text { em estudantes universitários brasileiros }\end{array}$ & $\begin{array}{l}\text { Bastos et al. } \\
(2014)^{20}\end{array}$ & $\begin{array}{l}\text { Cadernos de } \\
\text { Saúde Publica }\end{array}$ & Inglês \\
\hline 14 & Scopus & $\begin{array}{l}\text { Saúde mental e inatividade física durante a } \\
\text { gestação: Estudo transversal aninhado no } \\
\text { estudo de coorte BRISA }\end{array}$ & $\begin{array}{l}\text { Takahasi et } \\
\text { al. }(2013)^{21}\end{array}$ & $\begin{array}{l}\text { Cadernos de } \\
\text { Saúde Publica }\end{array}$ & Inglês. \\
\hline 13 & Scopus & $\begin{array}{l}\text { Uso de substâncias psicoativas, contexto } \\
\text { familiar e saúde mental em adolescentes } \\
\text { Brasileiros, Pesquisa nacional de Saúde dos } \\
\text { Escolares (PeNSE 2012) }\end{array}$ & $\begin{array}{l}\text { Malta et al. } \\
(2014)^{22}\end{array}$ & $\begin{array}{l}\text { Revista } \\
\text { Brasileira de } \\
\text { Epidemiologia }\end{array}$ & $\begin{array}{l}\text { Inglês e } \\
\text { Português }\end{array}$ \\
\hline 12 & Scopus & $\begin{array}{l}\text { Acessibilidade e resolubilidade da assistência } \\
\text { em saúde mental: A experiência do apoio } \\
\text { matricial }\end{array}$ & $\begin{array}{l}\text { Quinderé et } \\
\text { al. }(2013)^{23}\end{array}$ & $\begin{array}{l}\text { Ciência \& Saúde } \\
\text { Coletiva }\end{array}$ & Português \\
\hline 10 & $\begin{array}{l}\text { Web of } \\
\text { Science }\end{array}$ & $\begin{array}{l}\text { Transtorno mental comum na população } \\
\text { idosa: pesquisa de base populacional no } \\
\text { Município de Campinas, São Paulo, Brasil }\end{array}$ & $\begin{array}{l}\text { Borim et al. } \\
(2013)^{24}\end{array}$ & $\begin{array}{l}\text { Cadernos de } \\
\text { Saúde Pública }\end{array}$ & Português \\
\hline $\begin{array}{l}11(\mathrm{~S}) \\
10(\mathrm{WoS})\end{array}$ & $\begin{array}{l}\text { Scopus e } \\
\text { Web of } \\
\text { Science }\end{array}$ & $\begin{array}{l}\text { Apoio matricial em saúde mental na atenção } \\
\text { primária à saúde: uma análise da produção } \\
\text { científica e documental }\end{array}$ & $\begin{array}{l}\text { Bonfim et } \\
\text { al. }(2013)^{25}\end{array}$ & $\begin{array}{l}\text { Interface } \\
\text { (Botucatu) }\end{array}$ & Português \\
\hline $\begin{array}{l}6(\mathrm{~S}) \\
9(\mathrm{WoS})\end{array}$ & $\begin{array}{l}\text { Scopus e } \\
\text { Web of } \\
\text { Science }\end{array}$ & $\begin{array}{l}\text { A elaboração participativa de indicadores } \\
\text { para a avaliação em saúde mental }\end{array}$ & $\begin{array}{l}\text { Furtado et } \\
\text { al. }(2013)^{26}\end{array}$ & $\begin{array}{l}\text { Cadernos de } \\
\text { Saúde Pública }\end{array}$ & Português \\
\hline 8 & $\begin{array}{l}\text { Web of } \\
\text { Science }\end{array}$ & $\begin{array}{l}\text { Síndrome de Burnout em técnicos de } \\
\text { enfermagem de um hospital público do } \\
\text { Estado de São Paulo }\end{array}$ & $\begin{array}{l}\text { Ferreira } \\
\text { e Lucca } \\
(2015)^{27}\end{array}$ & $\begin{array}{l}\text { Revista } \\
\text { Brasileira de } \\
\text { Epidemiologia } \\
\end{array}$ & $\begin{array}{l}\text { Inglês e } \\
\text { Português }\end{array}$ \\
\hline 8 & $\begin{array}{l}\text { Web of } \\
\text { Science }\end{array}$ & $\begin{array}{l}\text { Prevalência de transtorno mental comum } \\
\text { na atenção primária }\end{array}$ & $\begin{array}{l}\text { Lucchese et } \\
\text { al. }(2014)^{28}\end{array}$ & $\begin{array}{l}\text { Acta Paulista de } \\
\text { Enfermagem }\end{array}$ & $\begin{array}{l}\text { Inglês e } \\
\text { Português }\end{array}$ \\
\hline 8 & $\begin{array}{l}\text { Web of } \\
\text { Science }\end{array}$ & $\begin{array}{l}\text { Bullying e fatores associados em } \\
\text { adolescentes brasileiros: análise da Pesquisa } \\
\text { Nacional de Saúde do Escolar (PeNSE 2012) }\end{array}$ & $\begin{array}{l}\text { Malta et al. } \\
(2014)^{29}\end{array}$ & $\begin{array}{l}\text { Revista } \\
\text { Brasileira de } \\
\text { Epidemiologia } \\
\end{array}$ & $\begin{array}{l}\text { Inglês e } \\
\text { Português }\end{array}$ \\
\hline $\begin{array}{l}8(\mathrm{WoS}) \\
5(\mathrm{~S})\end{array}$ & $\begin{array}{l}\text { Scopuse } \\
\text { Web of } \\
\text { Science }\end{array}$ & $\begin{array}{l}\text { Fatores de risco para o desenvolvimento de } \\
\text { crianças atendidas em Unidades de Saúde } \\
\text { da Família, ao final do primeiro ano de vida: } \\
\text { Aspectos sociodemográficos e de saúde } \\
\text { mental materna }\end{array}$ & $\begin{array}{l}\text { Ribeiro et } \\
\text { al. }(2014)^{30}\end{array}$ & $\begin{array}{l}\text { Ciência \& Saúde } \\
\text { Coletiva }\end{array}$ & Português \\
\hline 8 & $\begin{array}{l}\text { Web of } \\
\text { Science }\end{array}$ & $\begin{array}{l}\text { Família da pessoa com transtorno mental e } \\
\text { suas necessidades na assistência psiquiátrica }\end{array}$ & $\begin{array}{l}\text { Bessa et al. } \\
(2013)^{31}\end{array}$ & $\begin{array}{l}\text { Texto \& } \\
\text { Contexto - } \\
\text { Enfermagem } \\
\end{array}$ & $\begin{array}{l}\text { Inglês e } \\
\text { Português }\end{array}$ \\
\hline 7 & $\begin{array}{l}\text { Web of } \\
\text { Science }\end{array}$ & $\begin{array}{l}\text { Associações entre a prática de bullying } \\
\text { e variáveis individuais e de contexto na } \\
\text { perspectiva dos agressores }\end{array}$ & $\begin{array}{l}\text { Oliveira et } \\
\text { al. }(2016)^{32}\end{array}$ & $\begin{array}{l}\text { Jornal de } \\
\text { Pediatria }\end{array}$ & $\begin{array}{l}\text { Inglês e } \\
\text { Português }\end{array}$ \\
\hline 7 & $\begin{array}{l}\text { Web of } \\
\text { Science }\end{array}$ & $\begin{array}{l}\text { Teoria Motivacional do Coping: uma } \\
\text { proposta desenvolvimentista de análise do } \\
\text { enfrentamento do estresse }\end{array}$ & $\begin{array}{l}\text { Ramos et } \\
\text { al. }(2015)^{33}\end{array}$ & $\begin{array}{l}\text { Estudos de } \\
\text { Psicologia } \\
\text { (Campinas) } \\
\end{array}$ & Português \\
\hline 7 & $\begin{array}{l}\text { Web of } \\
\text { Science }\end{array}$ & $\begin{array}{l}\text { Condições de vida, gênero e saúde mental } \\
\text { entre trabalhadoras rurais assentadas }\end{array}$ & $\begin{array}{l}\text { Costa et al. } \\
(2014)^{34}\end{array}$ & \begin{tabular}{|l|} 
Estudos de \\
Psicologia (Natal) \\
\end{tabular} & Português \\
\hline $\begin{array}{l}7(\mathrm{WoS}) \\
5(\mathrm{~S})\end{array}$ & $\begin{array}{l}\text { Web of } \\
\text { Science e } \\
\text { Scopus }\end{array}$ & $\begin{array}{l}\text { Saúde mental e vulnerabilidade social: a } \\
\text { direção do tratamento }\end{array}$ & $\begin{array}{l}\text { Da Gama et } \\
\text { al. }(2014)^{35}\end{array}$ & $\begin{array}{l}\text { Revista Latino- } \\
\text { americana de } \\
\text { Psicopatologia } \\
\text { Fundamental }\end{array}$ & Português \\
\hline
\end{tabular}


Quadro 1. Caracterização dos estudos analisados.

\begin{tabular}{|c|c|c|c|c|c|}
\hline $\begin{array}{l}\text { No de } \\
\text { citações }\end{array}$ & $\begin{array}{l}\text { Base de } \\
\text { Dados }\end{array}$ & Título do artigo & Autor/Ano & Periódico & Idioma \\
\hline 7 & $\begin{array}{l}\text { Web of } \\
\text { Science }\end{array}$ & $\begin{array}{l}\text { Usuário de crack em situações de } \\
\text { tratamento: experiências, significados e } \\
\text { sentidos }\end{array}$ & $\begin{array}{l}\text { Paula et al. } \\
(2014)^{36}\end{array}$ & $\begin{array}{l}\text { Saúde e } \\
\text { Sociedade }\end{array}$ & $\begin{array}{l}\text { Inglês e } \\
\text { Português }\end{array}$ \\
\hline 7 & $\begin{array}{l}\text { Web of } \\
\text { Science }\end{array}$ & $\begin{array}{l}\text { Perfil sociodemográfico e clínico de } \\
\text { dependentes químicos em tratamento: } \\
\text { análise de prontuários }\end{array}$ & $\begin{array}{l}\text { Capistrano } \\
\text { et al. } \\
(2013)^{37}\end{array}$ & $\begin{array}{l}\text { Escola Anna } \\
\text { Nery }\end{array}$ & Português \\
\hline 7 & $\begin{array}{l}\text { Web of } \\
\text { Science }\end{array}$ & $\begin{array}{l}\text { Políticas sobre drogas no Brasil: a estratégia } \\
\text { de redução de danos }\end{array}$ & $\begin{array}{l}\text { Machado } \\
\text { e Boarini } \\
(2013)^{38} \\
\end{array}$ & $\begin{array}{l}\text { Psicologia: } \\
\text { Ciência e } \\
\text { Profissão } \\
\end{array}$ & Português \\
\hline 7 & Scopus & $\begin{array}{l}\text { Relação entre saúde mental e bem-estar } \\
\text { espiritual em pacientes de hemodiálise: Um } \\
\text { estudo correlacional }\end{array}$ & $\begin{array}{l}\text { Martínez e } \\
\text { Custódio } \\
(2014)^{39}\end{array}$ & $\begin{array}{l}\text { São Paulo } \\
\text { Medical Journal }\end{array}$ & Inglês \\
\hline 6 & Scopus & $\begin{array}{l}\text { A Gestão Autônoma da Medicação: Uma } \\
\text { intervenção analisadora de serviços em } \\
\text { saúde mental }\end{array}$ & $\begin{array}{l}\text { Onocko- } \\
\text { Campos et } \\
\text { al. }(2013)^{40} \\
\end{array}$ & $\begin{array}{l}\text { Ciência \& Saúde } \\
\text { Coletiva }\end{array}$ & Português \\
\hline 5 & Scopus & $\begin{array}{l}\text { "Fui lá no posto e o doutor me mandou foi } \\
\text { pra cá": Processo de medicamentalização } \\
\text { e (des)caminhos para o cuidado em saúde } \\
\text { mental na atenção primária }\end{array}$ & $\begin{array}{l}\text { Bezerra et } \\
\text { al. }(2014)^{41}\end{array}$ & $\begin{array}{l}\text { Interface } \\
\text { (Botucatu) }\end{array}$ & Português \\
\hline 5 & Scopus & $\begin{array}{l}\text { Narrativas no estudo das práticas em saúde } \\
\text { mental: Contribuições das perspectivas } \\
\text { de Paul Ricoeur, Walter Benjamim e da } \\
\text { antropologia médica }\end{array}$ & $\begin{array}{l}\text { Onocko- } \\
\text { Campos et } \\
\text { al. }(2013)^{42}\end{array}$ & $\begin{array}{l}\text { Ciência \& Saúde } \\
\text { Coletiva }\end{array}$ & Português \\
\hline 5 & Scopus & $\begin{array}{l}\text { Equipe de apoio e a construção coletiva do } \\
\text { trabalho em Saúde Mental junto à Estratégia } \\
\text { de Saúde da Família: Espaço de discussão e } \\
\text { de cuidado }\end{array}$ & $\begin{array}{l}\text { Prates et al. } \\
(2013)^{43}\end{array}$ & $\begin{array}{l}\text { Saúde e } \\
\text { Sociedade }\end{array}$ & Português \\
\hline 4 & Scopus & $\begin{array}{l}\text { O projeto terapêutico singular e as práticas } \\
\text { de saúde mental nos núcleos de apoio à } \\
\text { saúde da família (NASF) em Guarulhos } \\
\text { (SP), Brasil }\end{array}$ & \begin{tabular}{|l} 
Hori e \\
Nascimento \\
$(2014)^{44}$
\end{tabular} & $\begin{array}{l}\text { Ciência \& Saúde } \\
\text { Coletiva }\end{array}$ & Português \\
\hline 4 & Scopus & $\begin{array}{l}\text { Rede de Atenção Psicossocial: Qual o lugar } \\
\text { da saúde mental? }\end{array}$ & $\begin{array}{l}\text { Quinderé et } \\
\text { al. }(2014)^{45}\end{array}$ & Physis & Português \\
\hline 4 & Scopus & $\begin{array}{l}\text { Saúde mental de crianças e adolescentes } \\
\text { com epilepsia: Análise de fatores clínicos e } \\
\text { neuropsicológicos }\end{array}$ & $\begin{array}{l}\text { Moreira et } \\
\text { al. }(2014)^{46}\end{array}$ & $\begin{array}{l}\text { Arquivos de } \\
\text { Neuropsiquiatria }\end{array}$ & Inglês \\
\hline 4 & Scopus & $\begin{array}{l}\text { Políticas e práticas em saúde mental: As } \\
\text { evidências em questão }\end{array}$ & $\begin{array}{l}\text { Campos et } \\
\text { al. }(2013)^{47}\end{array}$ & $\begin{array}{l}\text { Ciência \& Saúde } \\
\text { Coletiva }\end{array}$ & Português \\
\hline 4 & Scopus & $\begin{array}{l}\text { Acesso à experiência em primeira pessoa na } \\
\text { pesquisa em Saúde Mental }\end{array}$ & $\begin{array}{l}\text { Leal e Serpa } \\
\text { Junior } \\
(2013)^{48}\end{array}$ & $\begin{array}{l}\text { Ciência \& Saúde } \\
\text { Coletiva }\end{array}$ & Português \\
\hline 4 & Scopus & $\begin{array}{l}\text { O Comitê Cidadão como estratégia } \\
\text { cogestiva em uma pesquisa participativa no } \\
\text { campo da saúde mental }\end{array}$ & $\begin{array}{l}\text { Passos et al. } \\
(2013)^{49}\end{array}$ & $\begin{array}{l}\text { Ciência \& Saúde } \\
\text { Coletiva }\end{array}$ & Português \\
\hline
\end{tabular}

Nota: A letra "S", entre parênteses, aponta para os trabalhos encontrados na base Scopus. As letras "WoS”, entre parênteses, apontam para os trabalhos encontrados na base Web of Science.

Fonte: Autoras.

Com relação ao contexto, foram analisadas as disciplinas ou bases teóricas de afiliação dos estudos e dos autores, sendo que Ciências Médicas/
Medicina, Psicologia e Saúde Coletiva foram as áreas que mais apareceram, dentre outras (Quadro 2). 
Quadro 2. Contextos dos estudos analisados seguido pela quantidade de trabalhos em que aparece, entre parênteses.

\begin{tabular}{|c|c|}
\hline Contextos nos & Ciências Médicas/Medicina $(\mathrm{n}=11)$ \\
\hline quais o conceito & Psicologia $(n=11)$ \\
\hline aparece & Saúde Coletiva $(\mathrm{n}=9)$ \\
\hline & Enfermagem $(\mathrm{n}=8)$ \\
\hline & Psiquiatria $(n=6)$ \\
\hline & Saúde Pública $(\mathrm{n}=6)$ \\
\hline & Neurologia/Neurociências $(n=2)$ \\
\hline & Vigilância em Saúde $(\mathrm{n}=2)$ \\
\hline & Saúde $(\mathrm{n}=2)$ \\
\hline & Epidemiologia $(n=1)$ \\
\hline & Psicanálise $(\mathrm{n}=1)$ \\
\hline & Ciências farmacêuticas $(\mathrm{n}=1)$ \\
\hline & Serviço social $(\mathrm{n}=1)$ \\
\hline
\end{tabular}

Nota: A letra "n" equivale a quantidade de trabalhos nos quais os termos aparecem.

Fonte: Autoras.

Os termos substitutos foram compreendidos como palavras e expressões que constantemente se repetiam no texto para se referir à saúde mental. Desse modo, Transtornos Mentais (Transtornos Mentais comuns, Transtornos Psiquiátricos) foram os termos mais citados, dentre outros (Quadro 3).

Os atributos foram entendidos como as palavras usadas para descrever e caracterizar o conceito. Aspectos psicossociais e descrições de sintomas psiquiátricos foram os atributos mais citados, dentre outros (Quadro 3).

Com relação aos antecedentes foram considerados os episódios e cenários históricos e contextuais que deram base para o conceito e se mostraram como condição antecedente para pesquisa. A questão da Reforma Psiquiátrica e da Luta Antimanicomial foram as temáticas mais abordadas (Quadro 3).

Os eventos consequentes se referiam aos episódios que resultaram do uso do conceito no trabalho pesquisado. Apareceram com mais frequência os Fatores de impacto na saúde mental (Quadro 3).

As Implicações e Hipóteses são uma forma de leitura da pesquisa que evita uma abordagem conclusiva e estática, trazendo indagações e possibilidades de pesquisas futuras. Nos textos pesquisados, a maioria dos artigos identificou fatores de impacto que tinham potencial para proteger a saúde mental (Quadro 3).

\section{Discussão}

Percebeu-se que muitas áreas do conhecimento publicam sobre saúde mental, sendo esse um tema que é transversal a várias disciplinas e, portanto, sofre influência de todas essas matrizes de pensamento. Apenas um artigo, intitulado "Saúde mental e vulnerabilidade social: A direção do tratamento" ${ }^{35}$ se preocupou abertamente com a conceituação de saúde mental. Nesse, os autores fizeram uma crítica ao conceito desenvolvido pela OMS, dialogando sobre saúde e normalidade.

De modo geral, perceberam-se duas dimensões que se associaram frequentemente ao uso do termo saúde mental em todas as categorias utilizadas, foram elas: 1) a psiquiatria e os transtornos mentais; e 2) a reforma psiquiátrica, os serviços de atenção psicossocial e a abordagem existencial da saúde mental enquanto sofrimento psíquico.

Essas dimensões citadas estão associadas a paradigmas em saúde mental, que de modo histórico e político, se consolidaram com relação aos saberes e práticas no campo da saúde. Paradigmas podem ser entendidos como modelos de explicação que oferecem métodos, hipóteses e soluções para problemas estudados pelos praticantes de uma ciência. São reconhecidos e aceitos universalmente até que entrem em crise, pela emergência de outro paradigma considerado mais adequado, de acordo com Thomas Khun ${ }^{50}$. Também Foucault ${ }^{51}$ coloca como o saber é construído e se articula em uma perspectiva histórica e não dada a priori, sendo engendrado a partir de uma constante disputa de poder e de forças.

Desse modo, entende-se que a Psiquiatria, enquanto um paradigma em saúde mental, vem se constituindo ao tomar para si a intervenção da loucura a partir do tratamento da doença mental, em um processo de institucionalização ocorrido em hospitais e manicômios ${ }^{2}$. Nesses espaços se desenvolveu uma psicopatologia que se organizou em torno da observação, descrição e classificação de sinais e sintomas, tendo como referência um ideal de normalidade.

Atualmente, a Associação Psiquiátrica Norte -Americana (APA) publica o Manual Diagnóstico e Estatístico de Transtornos Mentais (DSM) que é seguido e reconhecido mundialmente. Esse manual a-teórico auxilia profissionais a categorizarem os transtornos mentais mediante a presença de sintomas específicos e o tempo de sua ocorrência. A APA recomenda o uso do termo "transtorno mental" em detrimento do termo "doença mental", acreditando que a expressão implica 
Quadro 3. Termos atribuídos ao termo Saúde Mental seguido pela quantidade de trabalhos em que aparece, entre parênteses.

\begin{tabular}{|c|c|}
\hline \multirow{8}{*}{$\begin{array}{l}\text { Termos } \\
\text { substitutos } \\
\text { para o } \\
\text { conceito }\end{array}$} & $\begin{array}{l}\text { Transtornos Mentais/Transtornos Mentais Comuns/Transtornos Psiquiátricos ou } \\
\text { comportamentais }(\mathrm{n}=19)\end{array}$ \\
\hline & Indefinido - textos que utilizavam o termo sem substituí-lo $(\mathrm{n}=10)$ \\
\hline & Sofrimento psíquico ou sofrimento mental $(\mathrm{n}=7)$ \\
\hline & Doença psíquica, Doença mental ou Estado mental $(n=4)$ \\
\hline & Fenômeno ou condição psicossocial $(\mathrm{n}=2)$ \\
\hline & Loucura $(\mathrm{n}=1)$ \\
\hline & Estresse $(\mathrm{n}=1)$ \\
\hline & Uso de drogas tido como um transtorno mental $(\mathrm{n}=1)$ \\
\hline \multirow{5}{*}{$\begin{array}{l}\text { Atributos do } \\
\text { conceito }\end{array}$} & Aspectos psicossociais - aspectos relacionais, culturais, econômicos, comportamentais ( $\mathrm{n}=13$ ) \\
\hline & $\begin{array}{l}\text { Sintomas psiquiátricos - transtorno mental, adoecimento mental, medicação psiquiátrica } \\
(\mathrm{n}=12)\end{array}$ \\
\hline & Condições existenciais - sofrimento psíquico, questões subjetivas, angústia e mal-estar ( $\mathrm{n}=7$ ) \\
\hline & Uso de álcool e outras drogas $(n=5)$ \\
\hline & $\begin{array}{l}\text { Modelos de atenção à saúde mental - Usuários, Apoio matricial, Clínica ampliada, serviços } \\
\text { substitutivos }(\mathrm{n}=3)\end{array}$ \\
\hline \multirow{4}{*}{$\begin{array}{l}\text { Termos } \\
\text { Antecedentes }\end{array}$} & Reforma Psiquiátrica e luta antimanicomial $(\mathrm{n}=13)$ \\
\hline & Psiquiatria e os transtornos psiquiátricos $(\mathrm{n}=10)$ \\
\hline & Rede de atenção e políticas de saúde mental $(n=6)$ \\
\hline & Uso de álcool e outras drogas $(\mathrm{n}=2)$ \\
\hline \multirow{5}{*}{$\begin{array}{l}\text { Termos } \\
\text { Consequentes }\end{array}$} & Fatores de impacto na saúde mental $(\mathrm{n}=12)$ \\
\hline & Oferta de serviços substitutivos de assistência à saúde mental $(\mathrm{n}=4)$ \\
\hline & Fatores associados ao uso de drogas $(\mathrm{n}=3)$ \\
\hline & Autonomia e participação dos usuários de serviços de saúde mental (n=2) \\
\hline & novas metodologias de pesquisa em saúde mental $(n=2)$ \\
\hline \multirow{6}{*}{$\begin{array}{l}\text { Hipóteses e } \\
\text { Implicações }\end{array}$} & Identificar fatores de impacto que tinham potencial para proteger a saúde mental $(\mathrm{n}=16)$ \\
\hline & Considerar novos modelos de atenção à saúde mental $(\mathrm{n}=9)$ \\
\hline & Considerar novas metodologias de pesquisa em saúde mental $(\mathrm{n}=2)$ \\
\hline & $\begin{array}{l}\text { Considerar a dimensão política, epistemológica, jurídica, sociocultural e técnico-assistencial da } \\
\text { saúde mental }(\mathrm{n}=1)\end{array}$ \\
\hline & Considerar diferentes compreensões acerca do uso de drogas $(n=1)$ \\
\hline & Considerar associações entre saúde/doença mental e vulnerabilidade social $(\mathrm{n}=1)$ \\
\hline
\end{tabular}

Nota: A letra "n" equivale a quantidade de trabalhos nos quais os termos aparecem.

Fonte: Autoras.

uma definição ampla que agrega outras condições como a enfermidade mental, a incapacidade intelectual, os transtornos de personalidade e também o uso de álcool e outras drogas, embora não seja consenso no mundo inteiro ${ }^{52}$.

A Psiquiatria passou por um processo próprio de transformações históricas, conceituais e ideológicas. Mudanças importantes se destacam, pelo seu caráter preponderante, como a associação com as grandes companhias farmacêuticas e a busca pelos marcadores biológicos da etiologia dos distúrbios mentais ${ }^{53,54}$. A grande quantidade de trabalhos sustentados nesse paradigma não só comprovam a influência da disciplina na pesquisa em saúde mental, mas também o impacto dos medicamentos psicotrópicos no conhecimento produzido em psiquiatria, na economia e na assistência aos usuários em saúde mental.

Por outro lado, a Reforma Psiquiátrica engendrou movimentos que despontaram em vários países, como a Psiquiatria de Setor, a Psiquiatria Preventiva, a Antipsiquiatria, a Psicoterapia Institucional e a Psiquiatria Democrática, que são exemplos de como os movimentos da Reforma se manifestaram de diferentes formas pelo mundo ${ }^{55}$.

A Psiquiatria Democrática italiana, cujo representante foi Franco Baságlia, foi aquela que sobremaneira influenciou a reforma brasileira que se consolidou em 2001 com a Lei no 10.216, 
Lei da Reforma Psiquiátrica ${ }^{56}$. O referencial epistêmico da Psiquiatria Democrática questiona as definições de alienação, doença mental, anormalidade, isolamento, terapêutica e cura que vinham sendo difundidos pela psiquiatria clássica ${ }^{57}$.

A Psiquiatria Democrática reorientou o foco, antes na doença, para o sujeito, tomando como referência a ideia de redução fenomenológica de Edmund Husserl. A intenção desse movimento foi colocar a doença entre parêntesis e se ocupar da experiência do sujeito, sua dor, mal-estar e sofrimento $^{57}$. Destarte, o paradigma da Reforma Psiquiátrica muda o foco do diagnóstico e da doença mental para o sofrimento existencial do sujeito.

Esse paradigma também repensa os modelos assistenciais e os orienta para Atenção Psicossocial, que tenta fortalecer os vínculos sociais e comunitários, em vez de optar pela segregação e hospitalização $0^{58}$. Além disso, abre o campo de trabalho para uma atuação transdisciplinar, antes restrita a poucos especialistas, agora composta por diversos olhares profissionais, de modo que se forma um coletivo de agentes do cuidado, descentralizando a figura do médico ${ }^{58}$. É possível perceber essa descentralização também na pesquisa em Saúde Mental, dada a totalidade de áreas do conhecimento que produziram conteúdos relacionados ao tema (Quadro 2).

\section{Conclusões}

É perceptível o embate de forças entre a Psiquiatria e a Reforma Psiquiátrica, ainda hoje. Essa disputa de poder entre os dois paradigmas influência o modo como se conceitua e se compreende a saúde mental, influenciando por sua vez, as políticas públicas de assistência e as legislações de proteção aos usuários e a pesquisa científica. Além disso, há a questão da saúde mental ser percebida como um termo indefinido, já que alguns textos utilizaram a expressão saúde mental como se seu significado fosse presumido pela comunidade científica, sem ser necessário defini-la, confirmando também o pressuposto inicial de que a expressão é utilizada sem que sejam problematizados os discursos ou os paradigmas a ela associados.

\section{Colaboradores}

VP Alcântara trabalhou na concepção, pesquisa e metodologia. CAL Vieira trabalhou na concepção, discussão e redação final. SV Alves contribuiu com pesquisa e redação final. 


\section{Referências}

1. Canguilhem G. O Normal e o Patológico. $6^{\mathrm{a}}$ ed. rev. Rio de Janeiro: Forense Universitária; 2009.

2. Foucault M. História da loucura na idade clássica. 9a ed. São Paulo: Perspectiva; 2010.

3. Gaudenzi P. Normal e Patológico no naturalismo e no normativismo em saúde: a controvérsia entre Boorse e Nordenfelt. Physis 2016; 26(3):747-767.

4. Amarante P, Torre EHG. "De volta à cidade, sr. cidadão!” - reforma psiquiátrica e participação social: do isolamento institucional ao movimento antimanicomial. Rev Admnistr Publica 2018; 52(6):1090-1107.

5. Freitas F, Amarante P. Medicalização em psiquiatria. $2^{\mathrm{a}}$ ed. rev. Rio de Janeiro: Editora Fiocruz; 2017.

6. Andrade APM, Maluf SW. Experiências de desinstitucionalização na reforma psiquiátrica brasileira: uma abordagem de gênero. Interface (Botucatu) 2017; 21(63):811-821.

7. Amarante P, Lancetti A. Saúde mental e saúde coletiva. In: Campos GWS, Minayo MCS, Akerman M, Drumond Jr. M, Carvalho YM, organizadores. Tratado de saúde coletiva. São Paulo: Hucitec; 2006. p. 615-634.

8. Danziato L, Martins ACBL, Matos SS. Psicanálise e Biopolítica: o Fascínio do Discurso Médico. Rev Subjetiv 2018; (ed. esp.):44-54.

9. World Health Organization (WHO). International Classification of Functioning, Disability and Health: ICIDH-2. Geneva: WHO; 2001.

10. Canguilhem G. A saúde: conceito vulgar e questão filosófica. In: Canguilhem G. Escritos sobre a medicina. Rio de Janeiro: Forense Universitária; 2005. p. 35-48.

11. Almeida Filho N, Coelho MTA, Peres MFT. O Conceito de Saúde Mental. Rev USP 1999; 43:100-125.

12. Monteiro MCM, Holanda VR, Melo GP. Análise do conceito parto humanizado de acordo com o método evolucionário de Rodgers. Rev Enferm Centro-Oeste Mineiro 2017; 7:e1885.

13. Carbogim FC, Oliveira LB, Püschel VAA. Critical thinking: concept analysis from the perspective of Rodger's evolutionary method of concept analysis. Rev Latino-Am Enferm 2016; 24:e2785.

14. Van Noorden R, Maher BNR. The top 100 papers. $\mathrm{Na}$ ture 2014; 514(7524):550-553.

15. Ioannidis JPA, Boyack KW, Small H, Sorensen AA, Klavans $R$. Bibliometrics: Is your most cited work your best? Nature 2014; 514(7524):561-562.

16. Nassi-Calò L. Artigo investiga: seu trabalho mais citado é seu melhor trabalho? [Internet]. SciELO em Perspectiva; 2014 [acessado 2019 jul 19]. Disponível em: https://blog.scielo.org/blog/2014/11/24/artigo-investiga-seu-trabalho-mais-citado-e-seu-melhor-trabalho/\#.YZfZ5NDMLIU.

17. Nassi-Calò L. Declaração recomenda eliminar o uso do Fator de Impacto na Avaliação de Pesquisa [Internet]. SciELO em Perspectiva; 2013 [acessado 2019 jul 19]. Disponível em: https://blog.scielo.org/ blog/2013/07/16/declaracao-recomenda-eliminar-o -uso-do-fator-de-impacto-na-avaliacao-de-pesquisa/\#.YZfaU9DMLIU.
18. Bezerril MS, Chiavone FBT, Lima JVH, Vitor AF, Ferreira Júnior MA, Santos VEP. Ensino de enfermagem: uma análise do conceito segundo o método evolucionário de Rodgers. Esc Anna Nery 2018, 22(4):e20180076.

19. Gonçalves DA, Mari JJ, Bower P, Gask L, Dowrick C, Tófoli LF, Campos M, Portugal FB, Ballester D, Fortes S. Estudo multicêntrico brasileiro sobre transtornos mentais comuns na atenção primária: prevalência e fatores sociodemográficos relacionados. Cad Saude Publica 2014; 30(3):623-632.

20. Bastos JL, Barros AJD, Celeste RK, Paradies Y, Faerstein E. Discriminação de idade, classe e raça: Suas interações e associações com saúde mental em estudantes universitários brasileiros. Cad Saude Publica 2014; 30(1):175-186.

21. Takahasi EHM, Alves MTSSB, Alves GS, Del-Ben $\mathrm{CM}$, Barbieri MA. Saúde mental e inatividade física durante a gestação: Estudo transversal aninhado no estudo de coorte BRISA. Cad Saude Publica 2013; 29(8):1583-1594.

22. Malta DC, Oliveira-Campos M, Prado RR, Dias AJR, Bomtempo DB. Uso de substâncias psicoativas, contexto familiar e saúde mental em adolescentes Brasileiros, Pesquisa nacional de Saúde dos Escolares (PeNSE 2012). Rev Bras Epidemiol 2014; 17(Supl. 1):46-61.

23. Quinderé PHD, Jorge MSB, Nogueira MSL, Costa LFA, Vasconcelos MGF. Acessibilidade e resolubilidade da assistência em saúde mental: A experiência do apoio matricial. Cien Saude Colet 2013; 18(7):21572166.

24. Borim FSA, Barros MBA, Botega NJ. Transtorno mental comum na população idosa: pesquisa de base populacional no Município de Campinas, São Paulo, Brasil. Cad Saude Publica 2013; 29(7):1415-1426.

25. Bonfim IG, Bastos ENE, Góis CWL, Tófoli LF. Apoio matricial em saúde mental na atenção primária à saúde: uma análise da produção científica e documental. Interface (Botucatu) 2013; 17(45):287-300.

26. Furtado JP, Onocko-Campos RT, Moreira MIB, Trapé TL. A elaboração participativa de indicadores para a avaliação em saúde mental. Cad Saude Publica 2013; 29(1):102-110.

27. Ferreira NN, Lucca SR. Síndrome de burnout em técnicos de enfermagem de um hospital público do Estado de São Paulo. Rev Bras Epidemiol 2015; 18(1):6879.

28. Lucchese R, Sousa K, Bonfin SP, Vera I, Santana FR. Prevalência de transtorno mental comum na atenção primária. Acta Paul Enferm 2014; 27(3):200-207.

29. Malta DC, Prado RR, Dias AJR, Mello FCM, Silva MAI, Costa MR, Caiaffa WT. Bullying e fatores associados em adolescentes brasileiros: análise da Pesquisa Nacional de Saúde do Escolar (PeNSE 2012). Rev Bras Epidemiol 2014; 17(1):131-145.

30. Ribeiro DG, Perosa GB, Padovani FHP. Fatores de risco para o desenvolvimento de crianças atendidas em Unidades de Saúde da Família, ao final do primeiro ano de vida: Aspectos sociodemográficos e de saúde mental materna. Cien Saude Colet 2014; 19(1):215226. 
31. Bessa JB, Waidman MAP. Família da pessoa com transtorno mental e suas necessidades na assistência psiquiátrica. Texto Contexto Enferm 2013; 22(1):6170.

32. Oliveira WA, Silva MAI, Silva JL, Mello FCM, Prado RR, Malta DC. Associações entre a prática de bullying e variáveis individuais e de contexto na perspectiva dos agressores. J Pediatr 2016; 92(1):32-39.

33. Ramos FP, Enumo SRF, Paula KMP. Teoria Motivacional do Coping: uma proposta desenvolvimentista de análise do enfrentamento do estresse. Estud Psicol (Campinas) 2015; 32(2):269-279.

34. Costa MGSG, Dimenstein MDB, Leite JF. Condições de vida, gênero e saúde mental entre trabalhadoras rurais assentadas. Estud Psicol (Natal) 2014; 19(2):145-154.

35. Gama CAP, Campos RTO, Ferrer AL. Saúde mental e vulnerabilidade social: A direção do tratamento. Rev Latinoam Psicopatol Fundam 2014; 17(1):69-84.

36. Paula ML, Jorge MSB, Albuquerque RA, Queiroz LM. Usuário de crack em situações de tratamento: experiências, significados e sentidos. Saude Soc 2014; 23(1):118-130

37. Capistrano FC, Ferreira ACZ, Silva TL, Kalinke LP, Maftun FA. Perfil sociodemográfico e clínico de dependentes químicos em tratamento: análise de prontuários. Esc Anna Nery 2013; 17(2):234-241.

38. Machado LV, Boarini ML. Políticas sobre drogas no Brasil: a estratégia de redução de danos. Psicol Cien Prof 2013; 33(3):580-595.

39. Martínez BB, Custódio RP. Relação entre saúde mental e bem-estar espiritual em pacientes de hemodiálise: Um estudo correlacional. Sao Paulo Med J 2014; 132(1):23-27.

40. Onocko-Campos RT, Passos E, Palombini AL, Andrade PM, Borges LR. A Gestão Autônoma da Medicação: Uma intervenção analisadora de serviços em saúde mental. Cien Saude Colet 2013; 18(10):2889-2898.

41. Bezerra IC, Jorge MSB, Gondim APS, Lima LL, Vasconcelos MGF. "Fui lá no posto e o doutor me mandou foi pra cá": Processo de medicamentalização e (des) caminhos para o cuidado em saúde mental na atenção primária. Interface (Botucatu) 2014; 18(48):61-74.

42. Onocko-Campos RT, Palombini AL, Leal E, Diaz AG, Xavier MAZ. Narrativas no estudo das práticas em saúde mental: Contribuições das perspectivas de Paul Ricoeur, Walter Benjamim e da antropologia médica. Cien Saude Colet 2013; 18(10):2847-2857.

43. Prates MML, Garcia VG, Moreno DMFC. Equipe de apoio e a construção coletiva do trabalho em Saúde Mental junto à Estratégia de Saúde da Família: Espaço de discussão e de cuidado. Saude Soc 2013; 22(2):642652.

44. Hori AA, Nascimento AF. O projeto terapêutico singular e as práticas de saúde mental nos núcleos de apoio à saúde da família (NASF) em Guarulhos (SP), Brasil. Cien Saude Colet 2014; 19(8):3561-3571.

45. Quinderé PHD, Jorge MSB, Franco TB. Rede de Atenção Psicossocial: Qual o lugar da saúde mental? Physis 2014; 24(1):253-271.
46. Moreira FS, Lima AB, Fonseca PC, Maia-Filho HS Saúde mental de crianças e adolescentes com epilepsia: Análise de fatores clínicos e neuropsicológicos. Arq Neuro-Psiquiatr 2014; 72(8):613-618.

47. Campos GWS, Onocko-Campos RT, Del Barrio LR. Políticas e práticas em saúde mental: As evidências em questão. Cien Saude Colet 2013; 18(10):2797-2805.

48. Leal EM, Serpa Junior OD. Acesso à experiência em primeira pessoa na pesquisa em Saúde Mental. Cien Saude Colet 2013; 18(10):2939-2948.

49. Passos E, Otanari TMC, Emerich BF, Guerini L. O Comitê Cidadão como estratégia cogestiva em uma pesquisa participativa no campo da saúde mental. Cien Saude Colet 2013; 18(10):2919-2928.

50. Kuhn T. A estrutura das revoluções científicas. São Paulo: Perspectiva; 2000.

51. Foucault M. Microfísica do Poder. $6^{\circ}$ ed. Rio de Janeiro: Paz e Terra; 2017.

52. Pinheiro MA. O conceito de capital mental no campo da saúde mental no trabalho: uma análise crítica do discurso da organização mundial da saúde [tese] São Paulo: Fundação Getúlio Vargas; 2018.

53. Aguiar MP, Ortega FJG. Psiquiatria Biológica e Psicofarmacologia: a formação de uma rede tecnocientífica. Physis 2017; 27(4):889-910.

54. Assad ML, Ortega FJG. Usos de referências biológicas em publicações de Saúde Mental Global. Physis 2016; 26(4):1409-1428

55. Desviat M. A Reforma Psiquiátrica. $2^{\mathrm{a}}$ ed. Rio de Janeiro: Editora Fiocruz; 2015.

56. Amarante P. Saúde mental e atenção psicossocial. Rio de Janeiro: Fiocruz; 2007.

57. Amarante P. Reforma Psiquiátrica e Epistemologia. Cad Bras Saude Mental 2009; 1(1):34-41.

58. Yasui S. Rupturas e encontros: desafios da Reforma Psiquiátrica Brasileira [tese]. Rio de Janeiro: Fundação Oswaldo Cruz; 2006.

Artigo apresentado em 26/09/2018

Aprovado em 20/11/2020

Versão final apresentada em 22/11/2020

Editores-chefes: Romeu Gomes, Antônio Augusto Moura da Silva 
